\title{
Comparação Entre os Métodos PSO e Simulated Annealing na Minimização de Funções Não Lineares
}

\section{Comparison Between PSO and Simulated Annealing Methods in the Minimization of Nonlinear Functions}

Mateus Braga Oliveira ${ }^{1}$, Andressa Alves Machado da Silva², Joviana Sartori de Souza ${ }^{3}$

\section{RESUMO}

Este trabalho tem como objetivo a determinação de minimizadores globais de funções não lineares por meio do método de Otimização por Enxame de Partículas (PSO) e do método Simulated Annealing (SA). Os dois métodos estocásticos são comparados para verificação de sua eficiência na minimização de funções não lineares. São utilizadas funções não lineares de duas e cinco variáveis, com o objetivo de observar 0 comportamento dos métodos trabalhados para funções de diferentes dimensões. Também são feitas duas hibridizações, com a inserção do método da Busca Coordenada ao longo das iterações dos métodos PSO e Simulated Annealing. Assim, compara-se todos os métodos testados para as funções não lineares, verificando 0 tempo gasto na obtenção dos minimizadores globais das funções. A inserção da Busca Coordenada é feita para verificar a influência de uma busca nos eixos coordenados na localização dos mínimos globais. Deste modo, o foco deste trabalho é apresentar dois métodos de otimização e dois métodos híbridos para a determinação dos minimizadores globais para funções não lineares de várias variáveis. Assim, pode-se analisar a influência do método da Busca Coordenada, de acordo com sua inserção ao longo das iterações, no desempenho dos métodos PSO e SA.

Palavras-chave: Funções não lineares, Otimização por Enxame de Partículas (PSO), Recozimento Simulado (SA), Minimizadores globais, Busca Coordenada.

\section{ABSTRACT}

The objective of this work is the determination of global minimizers of nonlinear functions using the Particle Swarm Optimization (PSO) method and the Simulated Annealing (SA) method. The two stochastic methods are compared to verify their efficiency in minimizing nonlinear functions. Nonlinear functions of two and five variables are used, in order to observe the behavior of the methods worked for functions of different dimensions. Two hybridizations are also done, with the insertion of the Coordinate Search method over the iterations of the PSO and Simulated Annealing methods. Thus, all the methods tested for the non-linear functions are compared, checking the time taken to obtain the global function minimizers. The insertion of the Coordinate Search is done to verify the influence of a search in the coordinated axes in the location of the global minimums. Thus, the focus of this work is to present two optimization methods and two hybrid methods for the determination of global minimizers for nonlinear functions of several variables. Thus, one can analyze the influence of the Coordinate Search method, according to its insertion along the iterations, in the performance of the PSO and SA methods.

Keywords: Nonlinear Functions, PSO, Simulated Annealing, Global Minimizers, Coordinate Search.
${ }^{1}$ Mestre em Modelagem

Computacional na Universidade Federal Fluminense.

E-mail: mateus.broli@gmail.com

${ }^{2}$ Mestranda em Modelagem Computacional na Universidade Federal Fluminense, UFF/MCCT.

${ }^{3}$ Doutora em Modelagem Computacional. Professora adjunta da Universidade Federal Fluminense, Instituto do Noroeste Fluminense de Educação Superior 


\section{INTRODUÇÄAO}

Neste trabalho, serão comparados algoritmos de otimização na busca de minimizadores globais de funções não lineares de várias variáveis, com o objetivo de verificar qual dos métodos abordados é mais eficiente na resolução do problema proposto. Os métodos utilizados são o método PSO e o método Simulated Annealing. Além disso são utilizadas duas hibridizações, as hibridizações PSO/Busca Coordenada e Simulated Annealing/Busca Coordenada. Deste modo, o foco deste trabalho é apresentar métodos de otimização para a determinação dos minimizadores globais para funções não lineares. Com os resultados apresentados, será realizada uma comparação, verificando qual metodologia foi mais robusta na determinação dos pontos mínimos globais para os problemas testados. Assim, pode-se analisar a influência do método da Busca Coordenada no desempenho do método PSO e do método Simulated Annealing.

\subsection{Funções Não Lineares}

A partir de agora, apresentam-se as funções não lineares testadas. As funções estão presentes em BACK (1996) e MOLGA (2005). Foram realizados testes com funções de 2 e de 5 variáveis.

\section{Função Ackley}

A função Ackley é amplamente utilizada para testes de algoritmos de otimização. $\mathrm{Na}$ sua forma bidimensional, caracteriza-se por uma região externa quase plana, e um grande orifício no centro. A função representa um risco para algoritmos de otimização, particularmente algoritmos de subida, por ficar preso em um de seus muitos mínimos locais.

A função geralmente é avaliada no hipercubo $x_{i} \in[-32,78632,786]$, para todo $\mathrm{i}=1, \ldots, \mathrm{d}$, embora possa também ser restrito a um domínio menor.

$$
f(x)=-a \cdot \exp \left(-b \sqrt{\frac{1}{d} \sum_{1}^{d} x_{i}^{2}}\right)-\exp \left(-b \sqrt{\frac{1}{d} \sum_{1}^{d} \cos \left(c x_{i}\right)}\right)+a+\exp (1)
$$

onde $\mathrm{a}=20, \mathrm{~b}=0,2, c=2 \pi$ e d é a Dimensão da função.

O Mínimo global da função Ackley é: $f\left(x^{*}\right)=0 \mathrm{em} x=(0, \ldots, 0)$. 


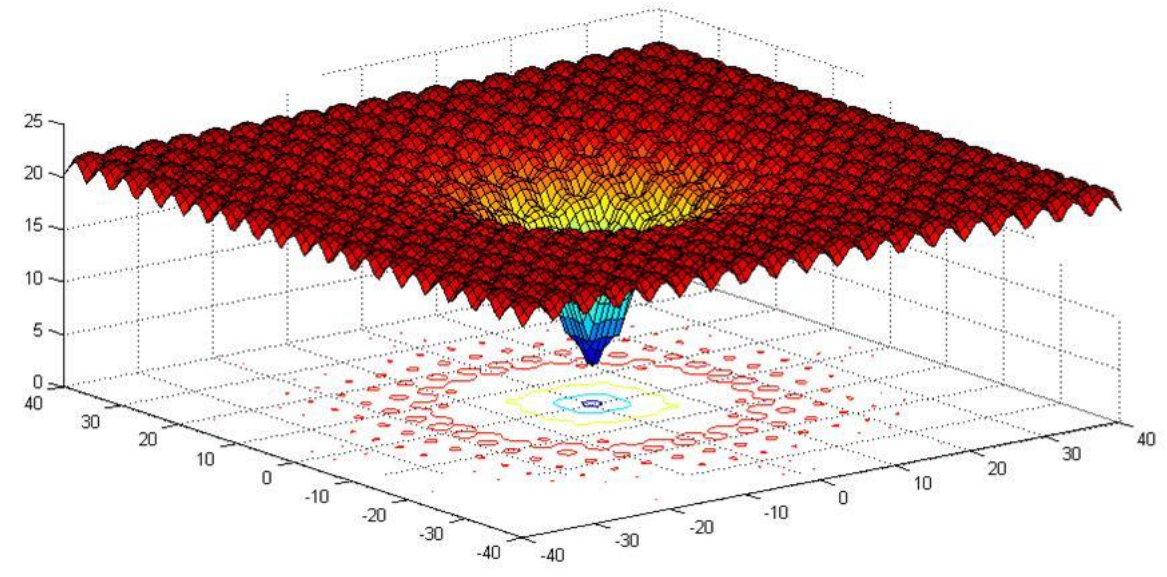

Figura 1. Função Ackley e seu mínimo global.

\section{Função Griewank}

Esta função possui muitos mínimos locais com distribuição regular. Possui d dimensões. Esta função é normalmente calculada na região $x_{i} \in[-600,600]$ para $\mathrm{i}=1, \ldots, \mathrm{d}$.

$$
f(x)=\sum_{i=1}^{d} \frac{x_{i}^{2}}{4000}-\prod_{i=1}^{d} \cos \left(\frac{x_{i}}{\sqrt{i}}\right)+1 .
$$

O Mínimo global da função Griewank é: $f\left(x^{*}\right)=0 \mathrm{em} x=(0, \ldots, 0)$. A função Griewank pode ser vista de diferentes modos, de acordo com a região que seu gráfico é feito.
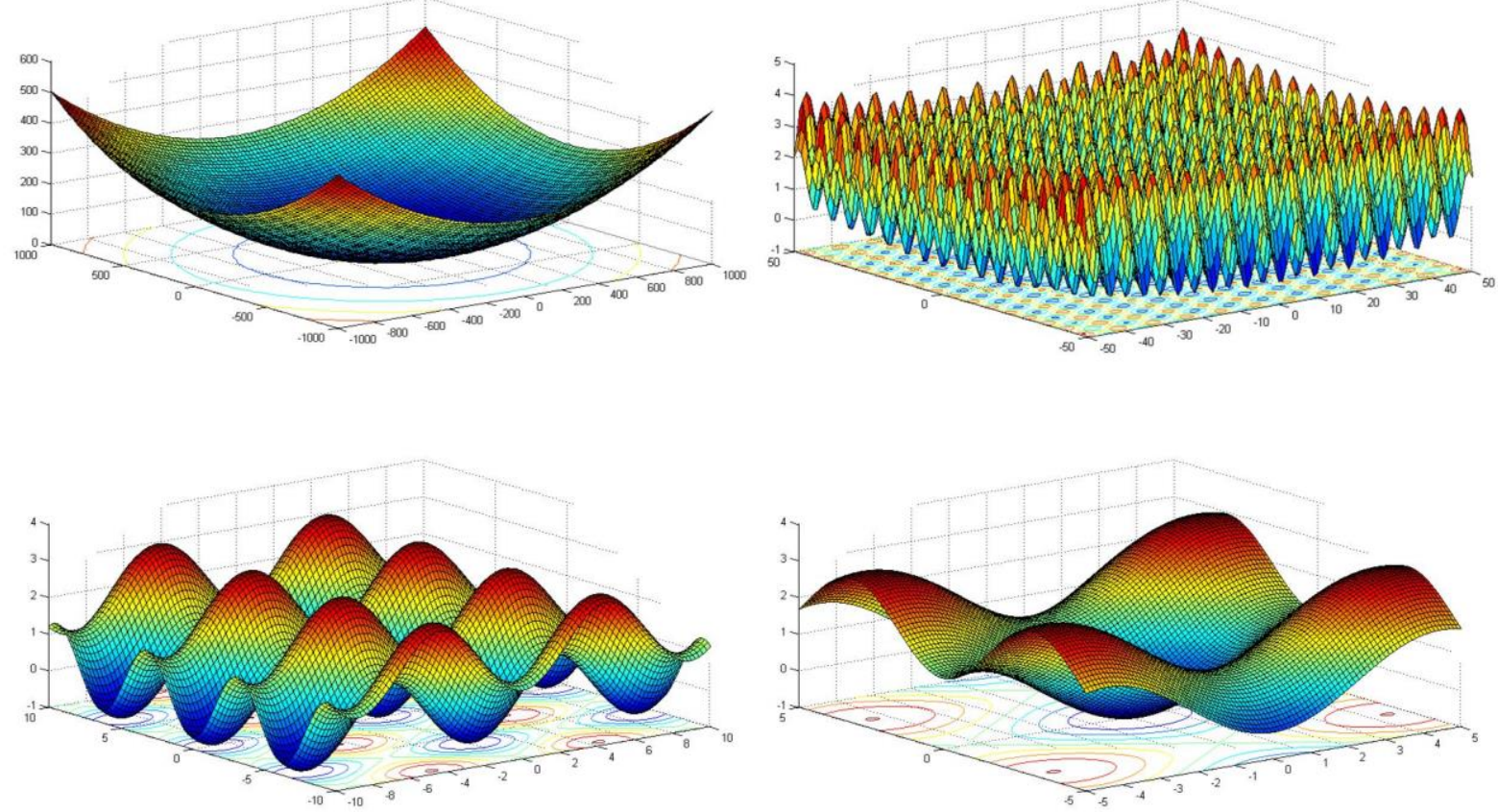

Figura 2. Função Griewank e suas variações. 


\section{Função Levy}

Nesta função foi considerada 20 e 30 dimensões. Ela é normalmente calculada na região $x_{i} \in[-10,10]$ para $\mathrm{i}=1, \ldots, \mathrm{d}$.

$f(x)=\operatorname{sen}^{2}\left(\pi \omega_{i}\right)+\sum_{i=1}^{d-1}\left(\omega_{i}-1\right)^{2}\left[1+10 \operatorname{sen}^{2}\left(\pi \omega_{i}+1\right)\right]+\left(\omega_{d}-1\right)^{2}\left[1+\operatorname{sen}^{2}\left(2 \pi \omega_{d}\right)\right]$,

onde $\omega_{i}=1+\frac{x_{i}-1}{4}$.

O Mínimo global da função Levy é dado por: $f\left(x^{*}\right)=0$ em $x=(1, \ldots, 1)$.

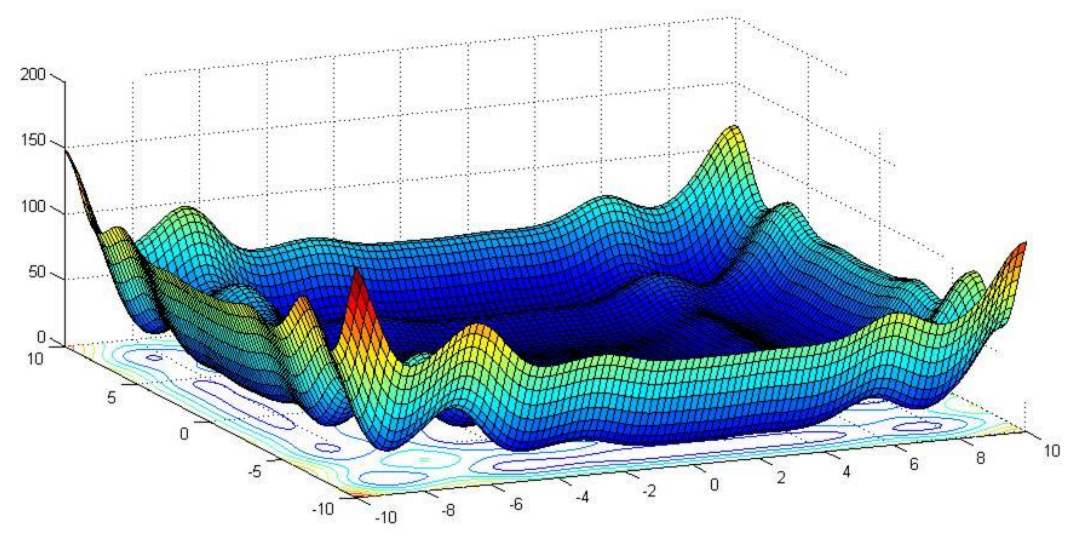

Figura 3. Função Levy e seu ponto de mínimo global.

\section{Função Rastrigin}

Esta função possui muitos mínimos locais. É altamente multimodal, mas as localizações são regularmente distribuídas. Calculada na região $x_{i} \in[-5.12,5.12]$ para $\mathrm{i}=1, \ldots, \mathrm{d}$.

$f(x)=10 d+\sum_{i=1}^{d}\left[x_{i}^{2}-10 \cos \left(2 \pi \cdot x_{i}\right)\right]$

O Mínimo global da função Rastrigin é: $f\left(x^{*}\right)=0 \mathrm{em} x=(0, \ldots, 0)$. A seguir, na Figura 4, é possível observar o gráfico da função Rastrigin. 


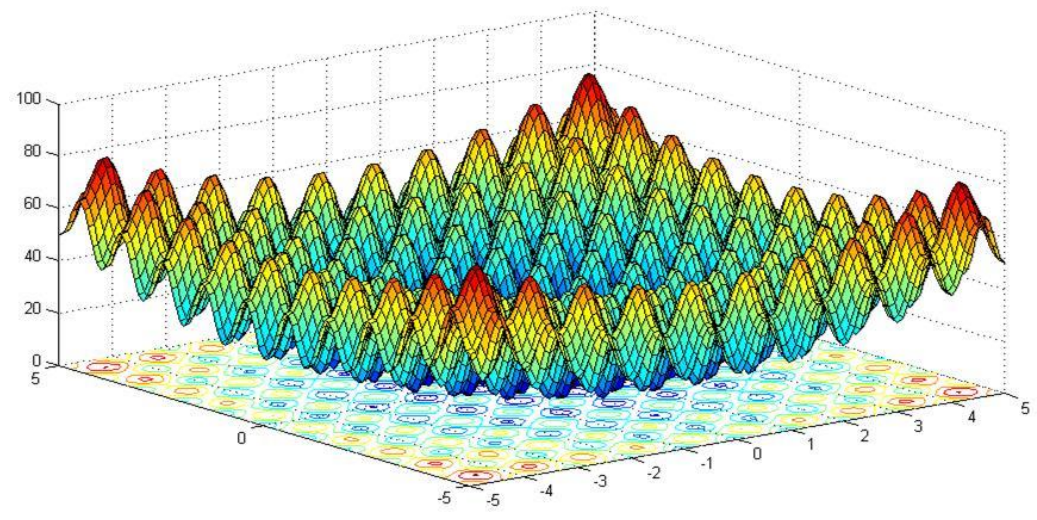

Figura 4. Função Rastrigin e seu minimizador global.

\section{Função Styblinski-Tang}

Esta função possui d dimensões. Neste trabalho, foram considerados $d=20$ e $d=30$. A função usualmente é calculada no hipercubo $x_{i} \in[-5,5]$ para $\mathrm{i}=1, \ldots, \mathrm{d}$.

$$
f(x)=\frac{1}{2} \sum_{i=1}^{d}\left(x_{i}^{4}-16 x_{i}^{2}+5 x_{i}\right)
$$

O Mínimo global é dado por: $f\left(x^{*}\right)=-39,16599 d$ em $x=(-2.903534, \ldots,-2.903534)$.

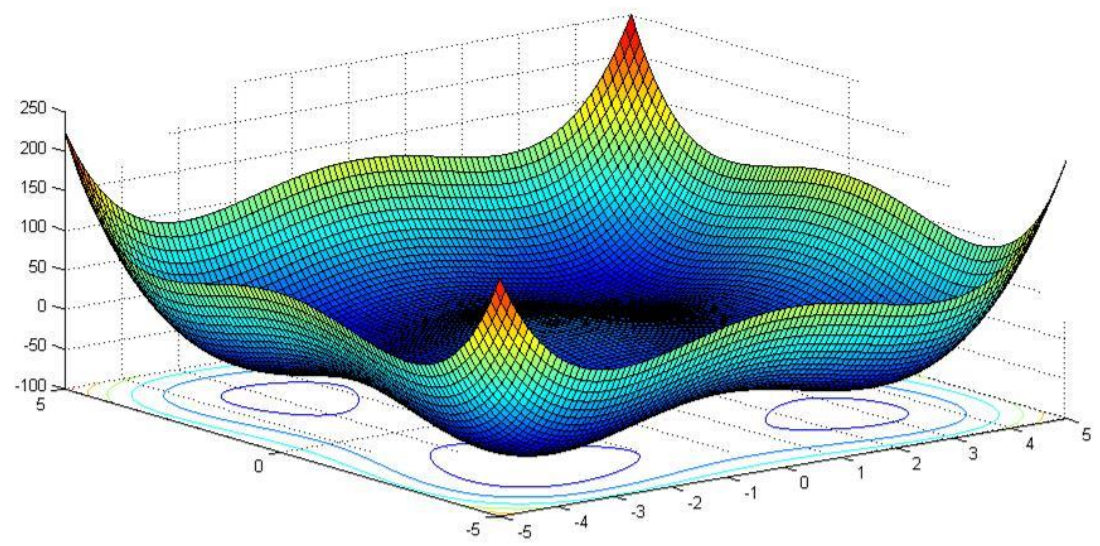

Figura 5. Função Styblinski-Tang.

\section{MATERIAIS E METODOS}

Foi utilizado neste trabalho, para a minimização das funções não lineares apresentadas, os seguintes métodos de otimização: O Método de Otimização por Enxame de Partículas (PSO) e o método Simulated Annealing (Recozimento Simulado - SA). Também mostra-se o método da Busca Coordenada, que foi utilizado na montagem de 
duas hibridizações a serem testadas no presente trabalho. A primeira hibridização acrescenta o método da Busca Coordenada (BC) em conjunto com o método PSO formando o método híbrido PSO/BC. O segundo método híbrido, parte do mesmo princípio, mas inclui o método da Busca Coordenada em conjunto com o método Simulated Annealing $(\mathrm{SA} / \mathrm{BC})$.

\subsection{Método PSO}

O método de otimização estocástica Enxame de Partículas (PSO) foi desenvolvido por EBERHART e KENNEDY (1995), e baseia-se em uma população, o enxame de $s$ partículas. A cada partícula associa-se um deslocamento, que no contexto heurístico, simboliza o movimento da partícula. A nova posição da i-ésima partícula no instante t+1 é representada por:

$$
x^{i}(t+1)=x^{i}(t)+v^{i}(t+1),
$$

isto é, tal posição é calculada adicionando-se à antiga posição $x^{i}(t)$ o vetor deslocamento $v^{i}(t+1)$.

Seguindo a versão do PSO usada por VAZ e VICENTE (2007), para cada partícula i, os componentes do vetor $v^{i}=\left(v_{1}^{i}, \ldots, v_{n}^{i}\right)^{T} \in R^{n}$ são atualizados pela equação escalar

$$
v_{j}^{i}(t+1)=l(t) v_{j}^{i}(t)+\mu(t) \omega_{1 j}(t)\left(y_{j}^{i}(t)-x_{j}^{i}(t)\right)+v \omega_{2 j}(t)\left(\hat{y}_{j}(t)-x_{j}^{i}(t)\right), j=1, \ldots, n
$$

Sendo $\imath(t)$ o fator de inércia, os parâmetros $\mu$ e $v$ números reais positivos chamados de parâmetro cognitivo e parâmetro social, respectivamente. Para todo $j=1, \ldots, n$, os números $\omega_{1 j}(t)$ e $\omega_{2 j}(t)$ são números aleatórios gerados de maneira uniforme no intervalo $(0,1)$. O valor $y_{j}^{i}(t)$ é o componente j do vetor posição $y^{i}(t)$ ocupado pela partícula com o i-ésimo melhor valor da função objetivo calculado antes. Finalmente, $\hat{y}_{j}(t)$ é o componente j do vetor posição $\hat{y}(t)$ ocupado pela partícula que possui o menor valor da função objetivo, dentre as s partículas.

A posição $\hat{y}(t)$ pode ser descrita como

$$
\hat{y}(t) \in \arg \min f(z), \text { onde } z \in\left\{y^{1}, \ldots, y^{s}(t)\right\}
$$

A seguir, o algoritmo do método PSO é mostrado na Figura 6. 


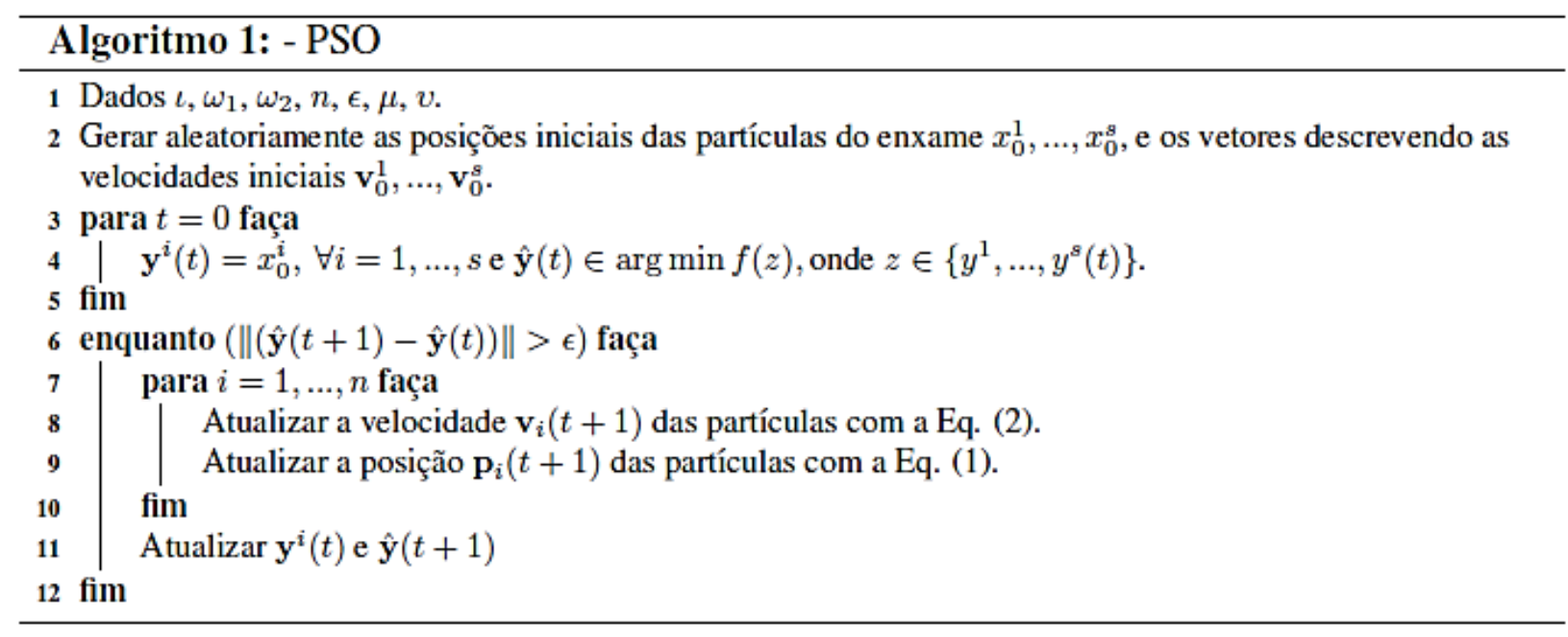

Figura 6. Algoritmo do Método PSO.

\subsection{Método Simulated Annealing}

O Método Simulated Annealing (Recozimento Simulado) (SA), foi um método proposto por KIRKPATRICK et al. (1983). Este método simula o recozimento de metais, fazendo assim uma analogia termodinâmica. É descrito em COSTA (2012), que o princípio do método sugere que, quando ocorre o resfriamento rápido, o produto gerado terá maior energia interna, e em um resfriamento lento, esta energia interna do produto gerado será menor. Assim, com uma redução rápida de temperatura, os produtos gerados são metaestáveis, e com uma redução lenta de temperatura, os produtos são mais estáveis e estruturalmente fortes.

Portanto, o método Simulated Annealing funciona do seguinte modo: emprega-se o Algoritmo de METROPOLIS (1953) em uma sequência de temperaturas decrescentes para gerar soluções de um problema de otimização; inicialmente, o método inicia-se com uma temperatura elevada, e a cada temperatura, geram-se soluções até que se alcance o equilíbrio àquela temperatura; a temperatura é então rebaixada e o processo prossegue até que não se obtenha mais uma diminuição de custo. A sequência de temperaturas empregada, juntamente com o número de iterações a cada temperatura, constitui uma prescrição de Annealing que deve ser definida empiricamente. Assim, o método Simulated Annealing permite a aceitação de soluções de piora, quando se tem temperaturas mais altas. Este fator auxilia para que o método não se prenda a minimizadores locais de uma função.

O algoritmo a seguir ilustra a fundamentação do Método Simulated Annealing: 


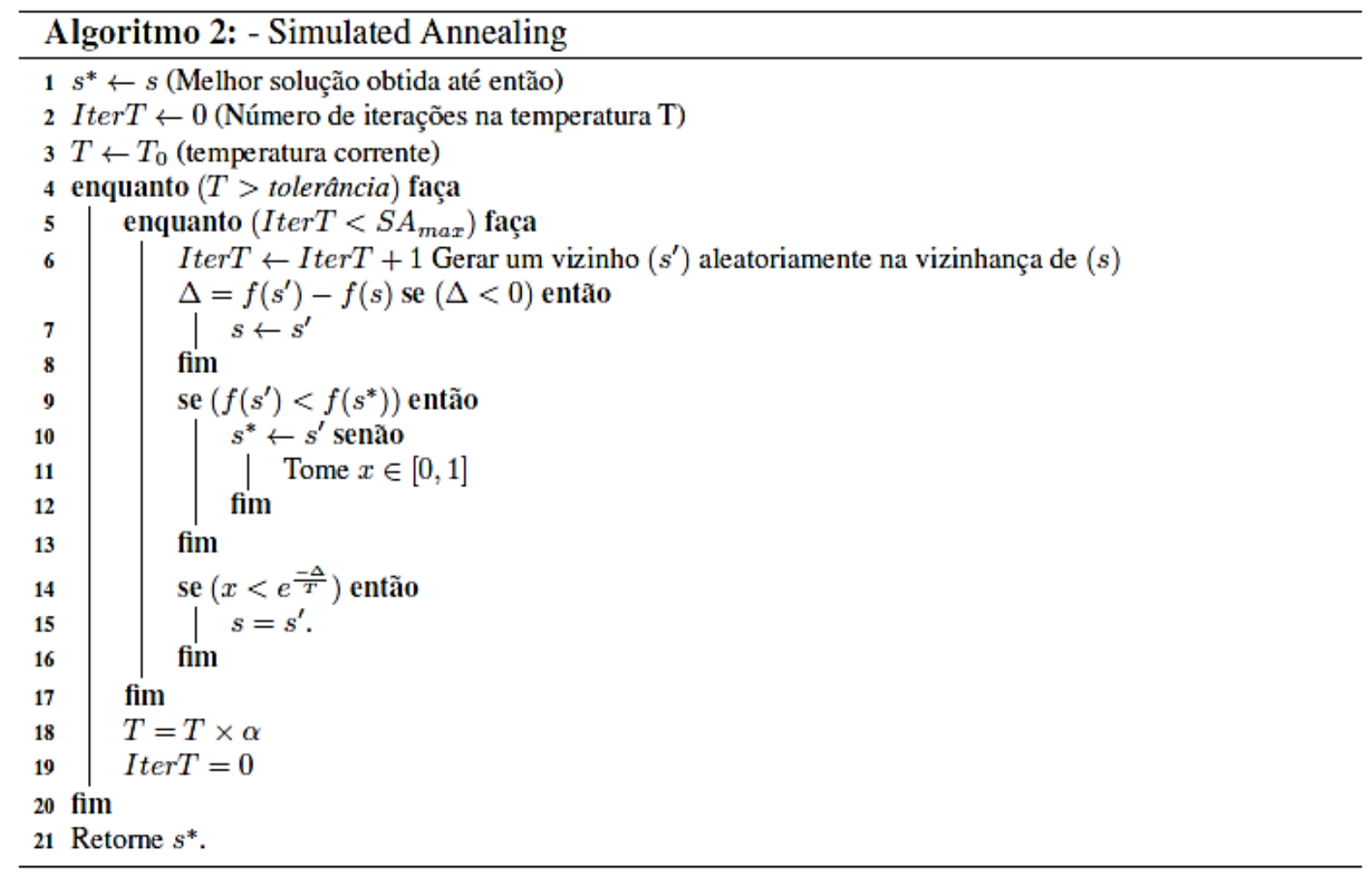

Figura 7. Algoritmo do Método Simulated Annealing.

\subsection{Método da Busca Coordenada}

O método da Busca Coordenada é um método de busca direta. Este método pode ser utilizado para resolver problemas de otimização. Uma característica dos métodos de busca direta, como o método da Busca Coordenada, é o fato dele não utilizar derivadas em seus cálculos, e também utilizar somente informações da função objetivo, na verificação do melhor ponto obtido.

Exemplificando, para funções reais de duas variáveis, segue-se o procedimento a seguir: efetua-se a busca nas direções paralelas aos eixos coordenados, ou seja, utilizando pontos ao norte, sul, leste e oeste do valor inicial. 


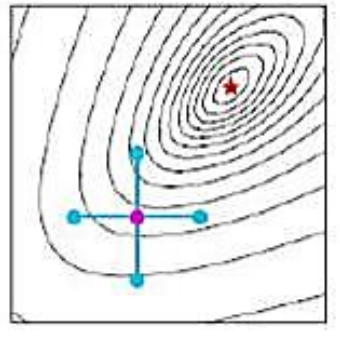

(a) Solução inicial

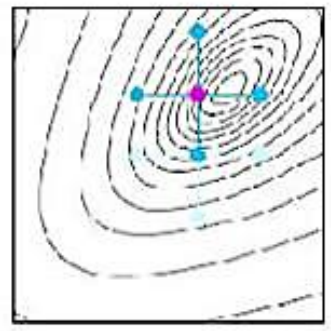

(d) Movimento para norte

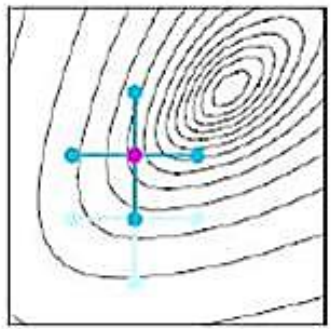

(b) Movimento para norte

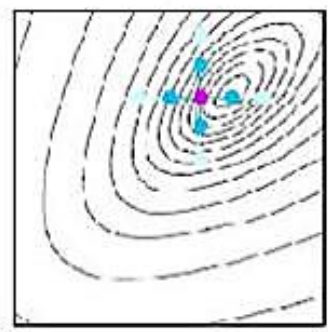

(e) Contração

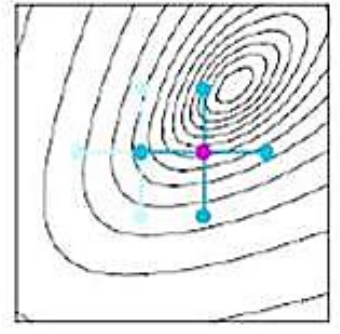

(c) Movimento para oeste

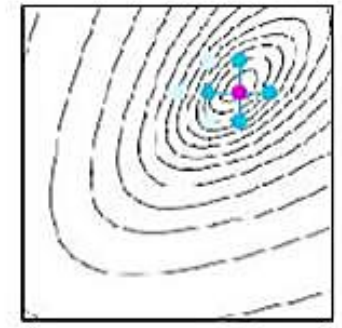

(f) Movimento para oeste

Figura 8. Passos do Método da Busca Coordenada.

De DINIZ-EHRHARDT et al. (2010), temos um algoritmo para o Método da Busca Coordenada:

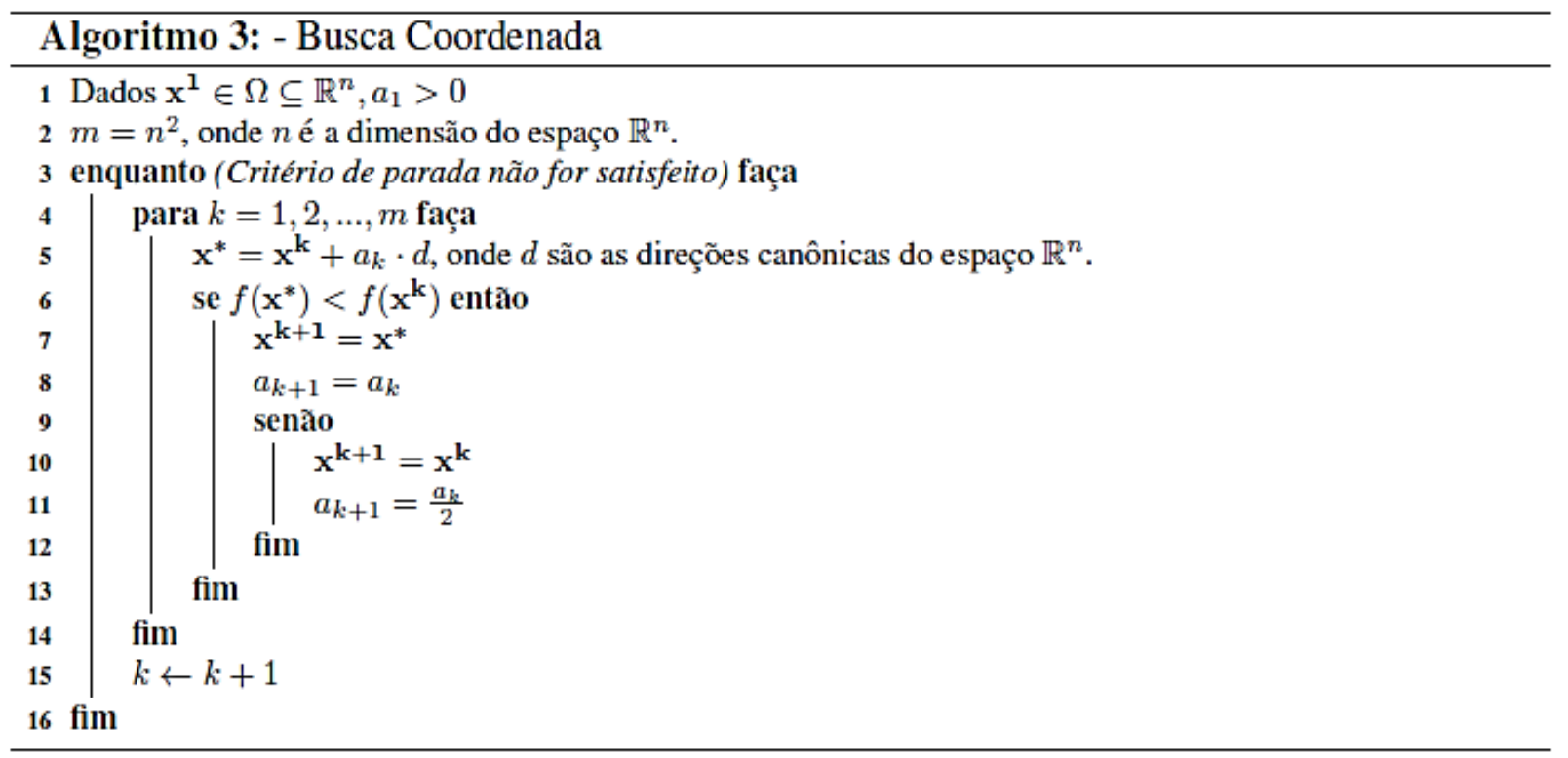

Figura 8. Algoritmo do Método da Busca Coordenada.

\subsection{Métodos Híbridos de Otimização}

Os métodos híbridos aqui testados, acrescenta-se o método da Busca Coordenada ao longo das iterações dos métodos PSO e Simulated Annealing. 


\section{Método PSO/Busca Coordenada}

O algoritmo PSO - Busca Coordenada (PSO/BC) é um algoritmo que adiciona o método da Busca Coordenada ao fim das iterações do algoritmo PSO. Realizam-se os passos do algoritmo PSO e encontra-se uma solução. Então é feito a busca coordenada nesta solução. Se a busca retornar uma melhor solução, o valor é atualizado e a função retorna ao PSO, até que a tolerância seja atendida, encontrando o minimizador da função. A busca coordenada é feita ao final de cada iteração do método PSO.

\section{Método Simulated Annealing/Busca Coordenada}

O algoritmo Simulated Annealing - Busca Coordenada (SA/BC) é um método híbrido que adiciona o método da Busca Coordenada ao fim das iterações do algoritmo Simulated Annealing. Esse método funciona da seguinte forma: realizam-se os primeiros passos do Simulated Annealing, quando uma melhor solução é encontrada, ele atualiza esse ponto e realiza uma busca coordenada ao seu redor, melhorando este novo ponto, ou não. Finalizada a busca coordenada, o método retorna para o SA e verifica se a solução encontrada satisfaz a tolerância desejada. Caso essa tolerância seja alcançada, o algoritmo é encerrado.

\section{RESULTADOS E DISCUSSAOO}

Agora, apresenta-se os resultados da execução dos métodos na determinação dos mínimos globais das funções não lineares aqui apresentadas. Os resultados trazem o tempo computacional que cada um dos métodos levou para obter o ponto de mínimo global de cada função. O tempo computacional refere-se ao tempo médio necessário para obtenção dos minimizadores globais, tendo sido realizadas 50 execuções de cada método. A tolerância utilizada como critério de parada foi o valor de $10^{-10}$. Assim, o mínimo obtido foi o valor do mínimo global, dentro desta tolerância desejada. Assim, as Tabelas com os resultados numéricos trazem uma aproximação para o valor do mínimo obtido.

Para uma melhor comparação, foi utilizado o mesmo gerador de número aleatórios para todos os métodos trabalhados. Assim, garantiu-se a obtenção dos mesmos resultados nas 50 execuções geradas. Mesmo modificando este gerador de números aleatórios, os resultados obtidos não sofreram variação. Logo, não apresenta-se aqui, os melhores e piores resultados de cada método, apenas o resultado que foi obtido em todas as execuções. 
A Tabela 1 traz os resultados para as funções não lineares, referentes ao tempo computacional para minimização das funções.

Tabela 1: Tempo computacional para obtenção dos mínimos globais das funções.

\begin{tabular}{c|c|c|c|c}
\hline Metodologia & SA & PSO & SA/BC & PSO/BC \\
\hline Funções & \multicolumn{3}{|c}{ Tempo Computacional (s) } \\
\hline Ackley - 2 variáveis & 0,0320 & 0,0320 & 0,0310 & 0,0310 \\
Ackley - 5 variáveis & 0,0780 & 0,2030 & 0,0620 & 0,2180 \\
Griewank - 2 variáveis & 0,0310 & 0,0320 & 0,0320 & 0,0310 \\
Griewank - 5 variáveis & 0,0680 & 0,1720 & 0,0600 & 0,1870 \\
Levy - 2 variáveis & 0,0470 & 0,0310 & 0,0310 & 0,0310 \\
Levy - 5 variáveis & 0,1090 & 0,3600 & 0,0780 & 0,3280 \\
Rastrigin - 2 variáveis & 0,0150 & 0,0160 & 0,0150 & 0,0160 \\
Rastrigin - 5 variáveis & 0,0630 & 0,1880 & 0,0470 & 0,1710 \\
Styblinski-Tang - 2 variáveis & 0,0310 & 0,0780 & 0,0310 & 0,0310 \\
Styblinski-Tang - 5 variáveis & 0,0780 & 0,2340 & 0,0620 & 0,2190 \\
\hline
\end{tabular}

Percebe-se, na Tabela 1, que o método Simulated Annealing e sua hibridização SA/BC foram eficientes, assim como o método PSO e a hibridização PSO/BC, pois todos os pontos de mínimo global das funções testadas foram obtidos. No entanto, o método SA obteve melhores resultados, consumindo um menor tempo computacional na resolução do problema proposto. A hibridização SA/BC teve ótimos resultados, melhores que o método PSO e sua hibridização com o método da Busca Coordenada.

Também foi analisado o número de iterações dos métodos trabalhados. A Tabela 2 mostra esses resultados.

Tabela 1: Tempo computacional para obtenção dos mínimos globais das funções.

\begin{tabular}{|c|c|c|c|c|}
\hline Metodologia & SA & PSO & SA/BC & PSO/BC \\
\hline Funções & \multicolumn{4}{|c|}{ Número de Iterações } \\
\hline Ackley - 2 variáveis & 91 & 20 & 86 & 25 \\
\hline Ackley - 5 variáveis & 97 & 116 & 93 & 113 \\
\hline Griewank - 2 variáveis & 92 & 23 & 90 & 22 \\
\hline Griewank - 5 variáveis & 95 & 114 & 94 & 114 \\
\hline Levy -2 variáveis & 95 & 21 & 93 & 20 \\
\hline Levy - 5 variáveis & 94 & 115 & 92 & 121 \\
\hline Rastrigin - 2 variáveis & 90 & 23 & 88 & 20 \\
\hline Rastrigin - 5 variáveis & 94 & 127 & 92 & 115 \\
\hline Styblinski-Tang - 2 variáveis & 91 & 23 & 88 & 26 \\
\hline Styblinski-Tang - 5 variáveis & 95 & 128 & 92 & 120 \\
\hline
\end{tabular}


Com os resultados da Tabela 2 vemos que as iterações têm pequenas variações em relação a cada método e sua hibridização com o método da Busca Coordenada. Ainda assim, destaca-se a eficiência de toda a metodologia proposta na resolução da minimização de funções não lineares. Todos os métodos obtiveram os mínimos globais das funções trabalhadas.

\section{CONSIDERAÇÓES FINAIS}

Verifica-se que os resultados numéricos para todas as funções foram encontrados, obtendo seus minimizadores globais. É possível perceber uma similaridade entre os métodos testados, ao se tratar de funções de duas variáveis. Para estas funções, os quatro métodos obtiveram os minimizadores globais das funções em um pequeno tempo. É importante destacar que todos os métodos encontraram todos os minimizadores globais das funções testadas, mostrando seu bom desempenho. É possível ver que as hibridizações foram superiores aos métodos testados, na maioria dos resultados obtidos, mostrando a boa influência do método da Busca Coordenada em conjunto com os métodos PSO e SA.

Assim, pode-se concluir que os métodos PSO e SA são eficientes na minimização de funções não lineares, assim como as hibridizações PSO/BC e SA/BC aqui mostradas. Estes métodos híbridos foram melhores, quando comparadas aos métodos não híbridos, tendo gasto um tempo menor na resolução do problema proposto.

\section{REFERENCIAS}

BACK, Thomas. Evolutionary algorithms in theory and practice: evolution strategies, evolutionary programming, genetic algorithms. Oxford university press, 1996.

COSTA, M. F. Aplicação de Meta-heurísticas no Escalonamento de Motoristas para o Transporte de Madeira. 2012. Dissertação de Mestrado. Universidade Federal do Espírito Santo.

DINIZ-EHRHARDT, Maria Aparecida; LOPES, Véra Lucia da Rocha; PEDROSO, Lucas Garcia. Métodos sem derivadas para minimização irrestrita. Notas em Matemática Aplicada, v. 49, n. 3, p. 87, 2010.

EBERHART, Russell; KENNEDY, James. A new optimizer using particle swarm theory. In: Micro Machine and Human Science, 1995. MHS'95., Proceedings of the Sixth International Symposium on. IEEE, 1995. p. 39-43.

KIRKPATRICK, Scott; GELATT, C. Daniel; VECCHI, Mario P. Optimization by simulated annealing. science, v. 220, n. 4598, p. 671-680, 1983. 
METROPOLIS, Nicholas et al. Equation of state calculations by fast computing machines. The journal of chemical physics, v. 21, n. 6, p. 1087-1092, 1953.

Molga, M., I\& Smutnicki, C. (2005), Test functions for optimization needs. Disponível em: <www.zsd.ict.pwr.wroc.pl/files/docs/functions.pdf.> Acesso em junho 2017.

SOUZA, J. S. Análise global da estabilidade termodinâmica de misturas: um estudo com o método do conjunto gerador. 2010. Tese de Doutorado. Tese de Doutorado, IPRJUerj.

VAZ, A. Ismael F.; VICENTE, Luís N. A particle swarm pattern search method for bound constrained global optimization. Journal of Global Optimization, v. 39, n. 2, p. 197-219, 2007. 